\title{
Anomalies Observed in VLF and LF Radio Signals on the Occasion of the Western Turkey Earthquake $\left(M_{w}=5.7\right)$ on May 19, 2011
}

\author{
Pier Francesco Biagi ${ }^{1}$, Flavia Righetti ${ }^{1}$, Tommaso Maggipinto ${ }^{1}$, Luigi Schiavulli ${ }^{1}$, Teresa Ligonzo ${ }^{1}$, \\ Anita Ermini ${ }^{2}$, Iren Adelina Moldovan ${ }^{3}$, Adrian Septimiu Moldovan ${ }^{4}$, Hugo Gonçalves Silva $^{5}$, \\ Mourad Bezzeghoud ${ }^{5}$, Michael E. Contadakis ${ }^{6}$, Dimitrios N. Arabelos ${ }^{6}$, \\ Thomas D. Xenos ${ }^{6}$, Aydin Buyuksarac ${ }^{7}$ \\ ${ }^{1}$ Department of Physics, University of Bari, Bari, Italy \\ ${ }^{2}$ Department of Industrial Engineering, University of Tor Vergata, Rome, Italy \\ ${ }^{3}$ National Institute of Earth's Physics, Seismological Department, Bucharest, Romania \\ ${ }^{4}$ AZEL-Designing Group S.R.L., Bucharest, Romania \\ ${ }^{5}$ Geophysical Centre of Évora and Physics Department, ECT, University of Évora, Évora, Portugal \\ ${ }^{6}$ Department of Surveying \& Geodesy, University of Thessaloniki, Thessaloniki, Greece \\ ${ }^{7}$ Department of Geophysics, Canakkale Onsekiz Mart University, Canakkale, Turkey \\ Email: pierfrancesco.biagi@uniba.it
}

Received May 30, 2012; revised July 4, 2012; accepted July 15, 2012

\begin{abstract}
Since 2009 a network of VLF $(20-60 \mathrm{kHz})$ and LF $(150-300 \mathrm{kHz})$ radio receivers is operating in Europe in order to study the disturbances produced by the earthquakes on the propagation of these signals. In 2011 the network was formed by nine receivers, of which three are located in Italy and one is in Austria, Greece, Portugal, Romania, Russia and Turkey. On May 19, 2001 an earthquake $\left(\mathrm{M}_{\mathrm{w}}=5.7\right)$ occurred in western Turkey, that is inside the "sensitive" area of the network. The radio data collected during April-May 2011 were studied using the Wavelet spectra, the Principal Component Analysis and the Standard Deviation trends as different methods of analysis. Evident anomalies were revealed both in the signals broadcasted by the TRT transmitter (180 kHz) located near Ankara and in a VLF signal coming from a transmitter located in Western Europe and collected by the receiver TUR of the network located in eastern Turkey. Evident precursor phases were pointed out. Some differences in the efficiency of the three analysis methods were revealed.
\end{abstract}

Keywords: Earthquake Precursors; European Radio Network; Radio Signal Analysis

\section{Introduction}

VLF radio signals lie in the $10-60 \mathrm{kHz}$ frequency band. These radio signals are used for worldwide navigation support, time signals and for military purposes. They are propagated in the earth-ionosphere wave-guide mode along great circle propagation paths. So, their propagation is strongly affected by the ionosphere conditions. LF signals lie in $150-300 \mathrm{kHz}$ frequency band. They are used for long way broadcasting by the few (this type of broadcasting is going into disuse) transmitters located in the world. These radio signals are characterized by the ground wave and the sky wave propagation modes [1]. The first generates a stable signal that propagates in the channel Earth-troposphere and is affected by the surface ground and troposphere condition. The second instead gives rise to a signal which varies greatly between day and night, and between summer and winter, and which propagates using the lower ionosphere as a reflector; its propagation is mainly affected by the ionosphere condition, particularly in the zone located in the middle of the transmitter-receiver path. The propagation of the VLF/LF radio signals is affected by different factors such as the meteorological condition, the solar bursts and the geomagnetic activity. At the same time, variations of some parameters in the ground, in the atmosphere and in the ionosphere occurring during the preparatory phase of earthquakes can produce disturbances in the above mentioned signals. As already reported by many previous studies [2-18] the disturbances are classified as anomalies and different methods of analysis as the residual $\mathrm{dA} /$ dP [15], the terminator time TT [9], the Wavelet spectra and the Principal Component Analysis have been used $[6,7]$.

Here the analysis carried out on LF and VLF radio 
signals using three different methods on the occasion of a strong earthquake occurred recently in Turkey is presented.

\section{The European Radio Network and the Turkey Earthquake}

Figure 1 shows the European VLF/LF Radio Network. The receivers are the Elettronika, the OmniPal and the MSK models described in [19,20]. The first ones can collect, with a sampling rate of $1 \mathrm{~min}$, the intensity of both VLF signals and LF signals (10 in total), the others the intensity and the phase of VLF signals (5 - 8 at least) with a sampling rate of $20 \mathrm{~s}$. All data from the various sites are collected on a weekly basis, at the Central Node of the Network located in the Department of Physics of University of Bari (Italy) and are processed for suitable analysis. In Figure 1 also the location of the transmitters, the signal of which is sampled by the various receivers, are indicated. Some peculiarities of these transmitters are reported in Table 1.

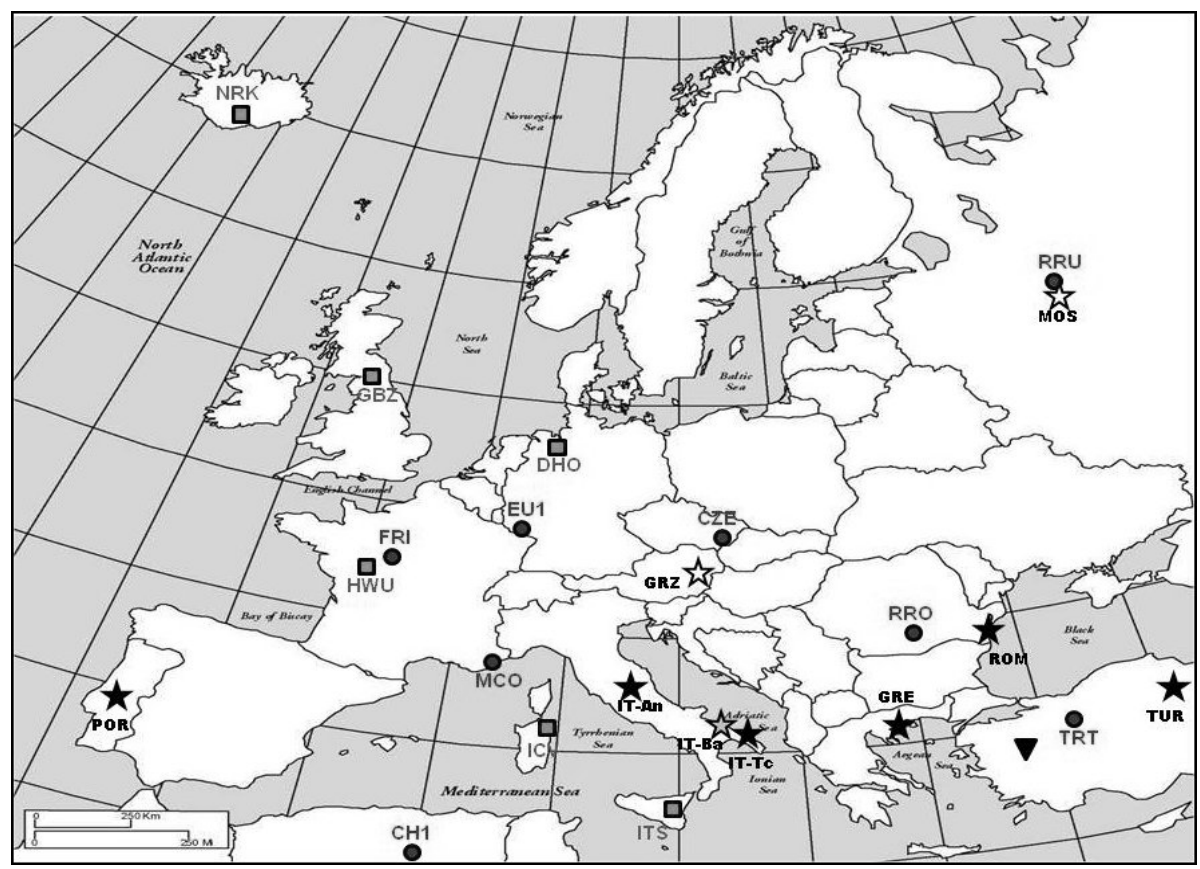

Figure 1. Map showing the different receivers and the VLF and LF transmitters of the European Radio Network. The stars show the location of the receivers (black $\equiv$ Elettronika, white $\equiv$ MSK, grey $\equiv$ OmniPal); the squares indicate the VLF transmitters and the circles the LF transmitters, the signals of which are collected by the different receivers. Some peculiarities of the transmitters are reported in Table 1 . The triangle indicates the epicentre of the western Turkey earthquake $(M=5.7)$ occurred on May 19, 2011.

Table 1. Peculiarities of the VLF and LF transmitters of the European radio network.

\begin{tabular}{|c|c|c|c|}
\hline Label & Country & Power (kW) & Frequency $(\mathrm{kHz})$ \\
\hline \multicolumn{4}{|c|}{ VLF transmitters } \\
\hline GBZ & United Kingdom & & 19.58 \\
\hline $\mathrm{ICV}$ & Sardinia, Italy & & 20.27 \\
\hline HWU & France & & 21.75 \\
\hline DHO & Germany & & 23.40 \\
\hline NRK & Iceland & & 37.50 \\
\hline ITS & Sicily, Italy & & 45.90 \\
\hline \multicolumn{4}{|c|}{ LF transmitters } \\
\hline RRO & Romania & 1200 & 153 \\
\hline FRI & France & 2000 & 162 \\
\hline TRT & Turkey & 1200 & 180 \\
\hline EU1 & Germany & 2000 & 183 \\
\hline $\mathrm{CH} 1$ & Algeria & 2000 & 198 \\
\hline $\mathrm{MCO}$ & France & 1200 & 216 \\
\hline RRU & Russia & 2500 & 261 \\
\hline $\mathrm{CZE}$ & Czech Republic & 500 & 270 \\
\hline
\end{tabular}


On May 19, 2011 an earthquake with magnitude $\mathrm{M}_{\mathrm{w}}=$ 5.7 struck in Simav, Kutahya (Turkey); the location is indicated in Figures 1 and 2. An intense aftershocks activity occurred for more than one month releasing an energy equivalent to an earthquake with the same magnitude of the main shock. The area belongs to one of the most important tectonic units of the Western Anatolia extension regime. In general, in this region the seismic activity occurs on the E-W tectonic line and on its branches and strong earthquakes are expected. The most intense and damaging recent earthquakes happened in 1928 with $\mathrm{M}$ (Richter) of 6.2 and in 1970 with $\mathrm{M}=7.2$.

In a recent study [21] the LF data collected during about two years by the receivers of the Network have been analysed. The earthquakes with $\mathrm{M}_{\mathrm{w}} \geq 5.0$ occurred in the same period, located in a $300 \mathrm{~km}$ radius around each receiver/transmitter and within the 5th Fresnel zone related to each transmitter-receiver path, have been selected. The Wavelet analysis was applied on the time series of the radio signals intensity and some anomalies related to the above mentioned earthquakes have been revealed.
In this framework, the epicentre of the mentioned Simav earthquake is inside the $300 \mathrm{~km}$ radius circle around the TRT $(180 \mathrm{kHz})$ transmitter, the signal of which is sampled by three receivers (GR, IT-An, IT-Tc) of the Network, as it is detailed in Figure 2(a).

With regard to the VLF radio signals, several results indicate that the area inside the 5th Fresnel zone defined by the transmitter and the receiver, is the most sensitive for the seismic disturbances on the radio propagation [13-15]. In this framework the Simav earthquakes is inside the 5 th Fresnel zone defined by the ITS $(45.9 \mathrm{kHz})$ transmitter and the TUR receiver of the Network, as shown in Figure 2(b).

Here the two previous situations have been investigated in details and different methods of analysis were used in order to reveal possible anomalies.

\section{Methods of Analysis}

In this study, the Wavelet spectra, the Standard Deviation trends and the Principal Component technique were used as different methods of analysis.
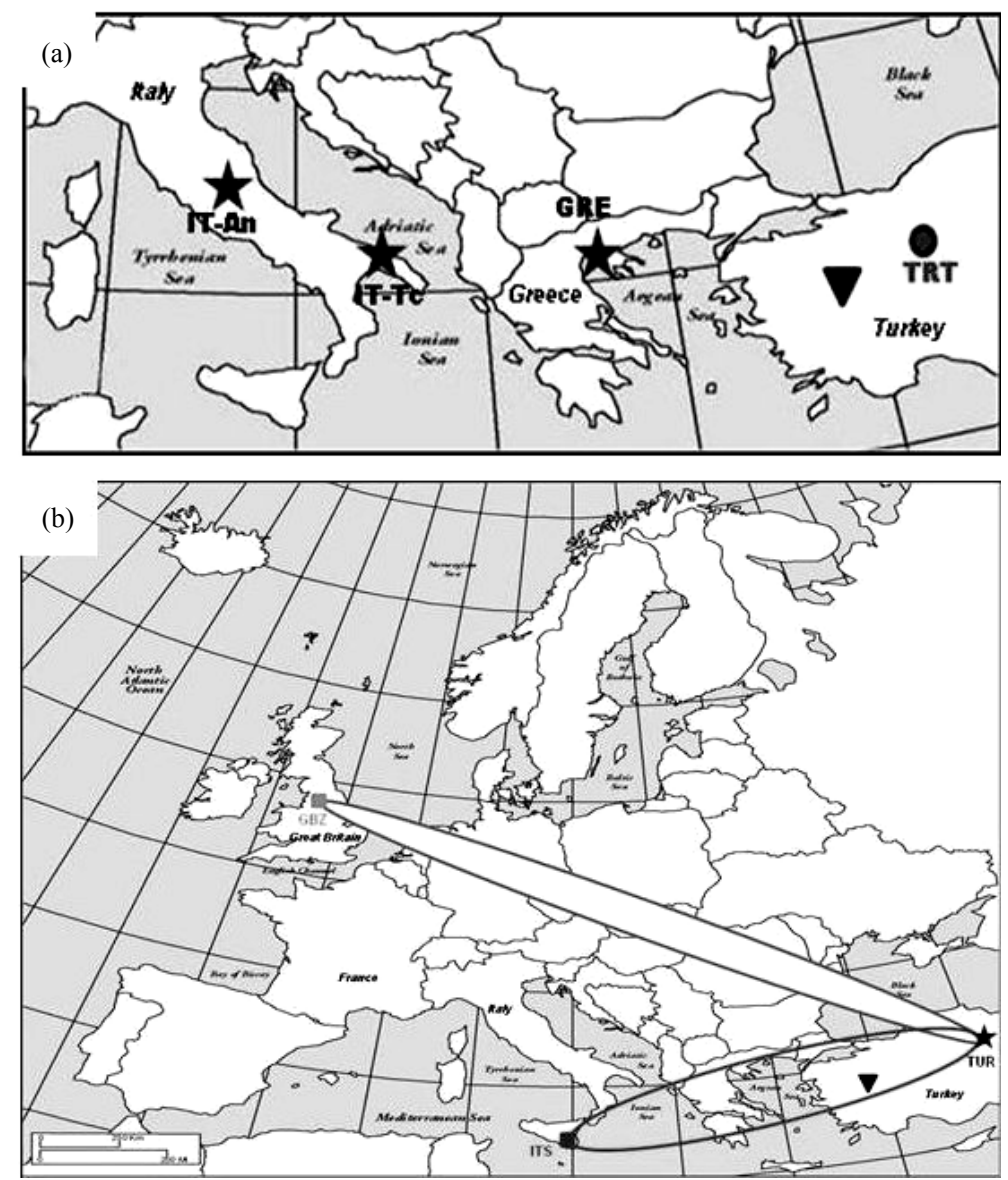

Figure 2. (a) Map showing the location of the receivers GR, IT-An and IT-Te recording the TRT (180 kHz) signal. The triangle indicates the epicentre of the earthquake; (b) Map of the 5th Fresnel zones defined by GBZ transmitter-TUR receiver and ITS transmitter-TUR receiver. The epicentre of the earthquake is indicated by a triangle. 


\subsection{Wavelet Analysis}

The Wavelet transform allows to highlight the spectral components of a signal by using variable-width time windows and by considering that the frequency content of these windows is in inverse relation to the time widths; so, the localization of the signal is simultaneously obtained both in time and in frequency [22,23]. In this study, we adopted as Wavelet the "Morlet function" [24]. In this case the Wavelet transform of a time signal is a complex series that can be usefully represented by its square amplitude, i.e. we consider the so-called Wavelet power spectrum. The power spectrum is a two dimensions plot that, once properly normalized with respect to the power of the white noise, gives information on the strength and precise time of occurrence of the various Fourier components which are present in the original time series. Generally, colour from blue to red indicates increase in the power strength; so, red zones define anomalies [7].

\subsection{Standard Deviation Trends}

For a sample of $\mathrm{n}$ data, the Standard Deviation $(S D)$ is calculated as follows:

$$
S D=\sqrt{\frac{\sum_{1}^{n}\left(x_{i}-x_{m}\right)^{2}}{n-1}}
$$

where $x_{m}$ is the mean value of the $x_{i}$ values. $S D$ shows how much variation or "dispersion" there is from the mean. A low $S D$ value indicates that the data points tend to be very close to the mean, whereas high $S D$ value indicates that the data points are spread out over a large range of values. The $S D$ can be calculated for each set of day-time data and night-time data and particularly low/high values define anomalies, that is low or high dispersion of the data. For a larger evidence we used the $\%$ value defined as $\frac{S D-S D_{m}}{S D_{m}}$ where $S D$ is the value of each day/night-time data and $S D_{m}$ is the mean value of the $S D$ data set in the whole time interval analyzed. The related trends are the Standard Deviation trends and the values over $\pm 2 \sigma$ (Standard Deviation) in these trends were assumed as low/high values defining an anomaly.

\subsection{Principal Component Analysis}

The Principal Component analysis (PCA) is a statistical technique whose purpose is to condense the information of a large set of correlated variables into a few variables (principal components), while not throwing overboard the variability present in the dataset [25]. The principal components are derived as a linear combination of the variables of the dataset, with weights chosen so that the principal components become mutually uncorrelated. Each component contains new information about the dataset and it is ordered so that the first few components account for most of the variability. The main result can be considered the plot that represents the first principal component (PC1) versus the second one (PC2); here, different colours of the points represent homogeneous groups and where outliers appear (a single point of different colour or a point with the same colour of an homogeneous group but standing far away from the group), these points define anomalies [7]. A number is reported near such points counting the days from the beginning of the data set under study.

\section{Data Analysis}

Generally, due to the different conditions of the ionosphere, the VLF radio signals are less disturbed during the night than during the day. For this reason, in the previous studies for revealing seismic anomalies, the analysis of the VLF radio data has been performed only on the night-time data. Here we have operated in the same way. As for the LF radio signals, on the basis of the previous results $[1-3,5]$ not only the night-time data but also the day-time ones have been examined.

As a first step, the radio data used in this study have been separated in day-time data and the night-time data. With regard to the VLF signals, we have selected different time ranges in order to obtain data related at proper night time conditions (basically related to darkness) along all the paths. As regard the LF signals, we selected the range from 8.00 to 13.00 (UT) for the day-time and the range from 20.00 to 22.00 (UT) for the night-time; this last choice is forced by the occurrence of an interruption of 3 - 4 hours in some radio broadcasts generally after the local 24.00 .

\section{Results and Discussion}

At first, the case reported in Figure 2(a) is considered. Figure 3 shows the results of the analysis of the TRT radio data collected at day-time by the GR, IT-An and IT-Tc receivers during April-May 2011. Top, middle and bottom of Figure 3 refer respectively to the Wavelet Spectra, the Standard Deviation trends and the PCA scatter plots. Both the Wavelet Spectra and the Standard Deviation trends reveal anomalies during the first fifteen days of April and the anomalies seem to be correlated; on the contrary the anomalies (referred to as numbered points in the scatter plot) revealed by the PCA analysis seem random. Figure 4 shows a similar analysis done on the nighttime data. Also in this case the indication of the Wavelet Spectra and of the Standard Deviation trends are well correlated, i.e. for all the receivers, some anomalies appear in the first fifteen days of April mainly in the period 
10 - 15 April; then a clear anomaly stands up in the data related to the GR receiver before the time occurrence of the Turkey earthquake. The indication of the PCA scatter plots sometimes agrees with the previous ones but also some disagreement can be revealed. For example the outliers 4 and 11 in IT-Tc data, the outlier 12 in IT-An data and the outlier 48 in GR data coincide with the anomalies emerging with the other analysis methods, whereas outliers 44 in IT-Tc, 44 and 57 in IT-An and the outlier 7 in GR data are not borne out by other results.

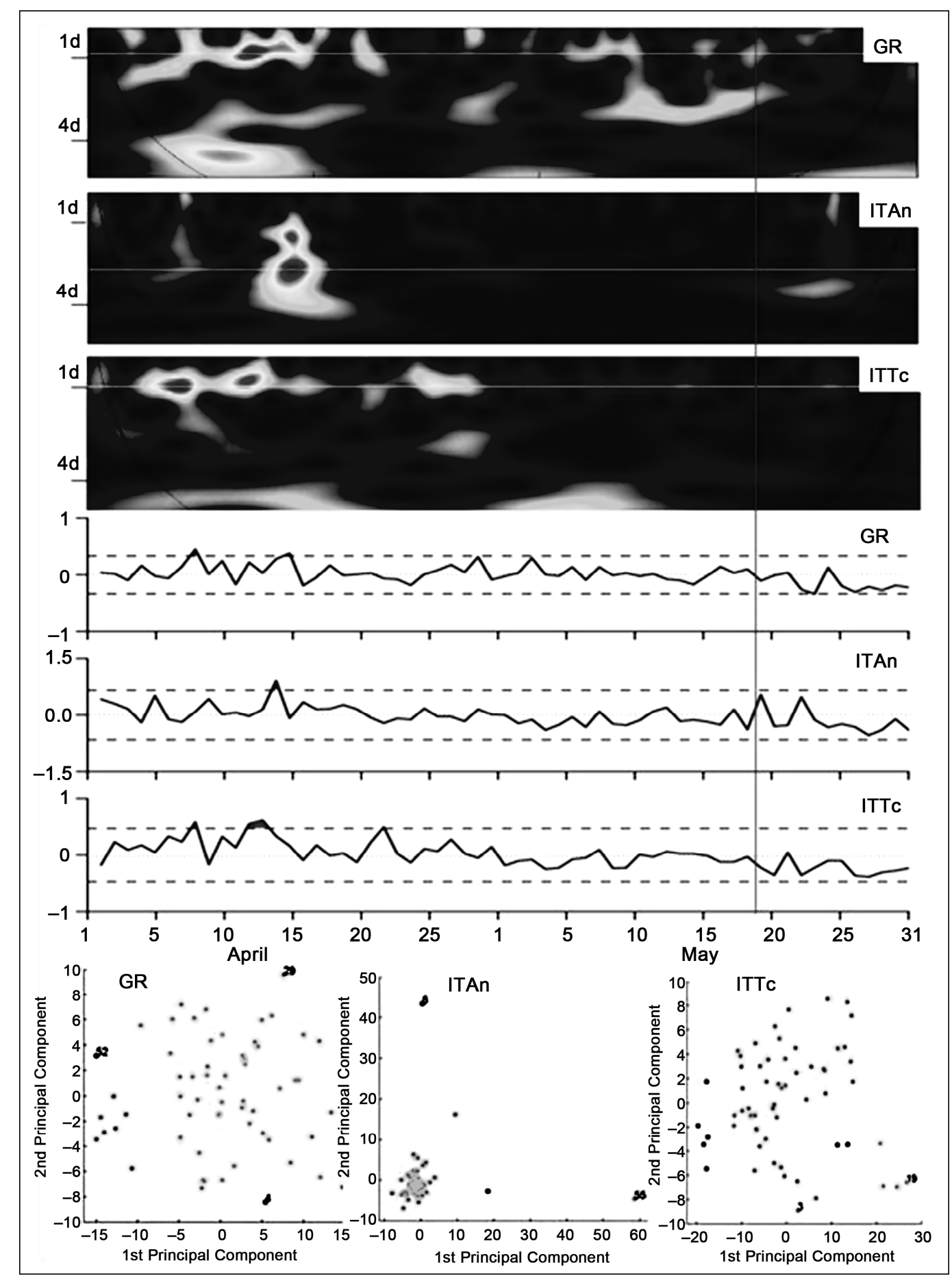

Figure 3. Results of the analysis of the TRT radio data collected by the GR, IT-An and IT-Te receivers during April-May 2011, at day-time. At the top the Wavelet spectra, at the middle the Standard Deviation trends and at the bottom the PCA plots. In the Standard Deviation trends the zones over $2 \sigma$ are filled in red; in the PCA plots the numbers on some point (possible outlier) denotes the day counted from April 1. The vertical red line indicates the occurrence of the Turkey earthquake. 


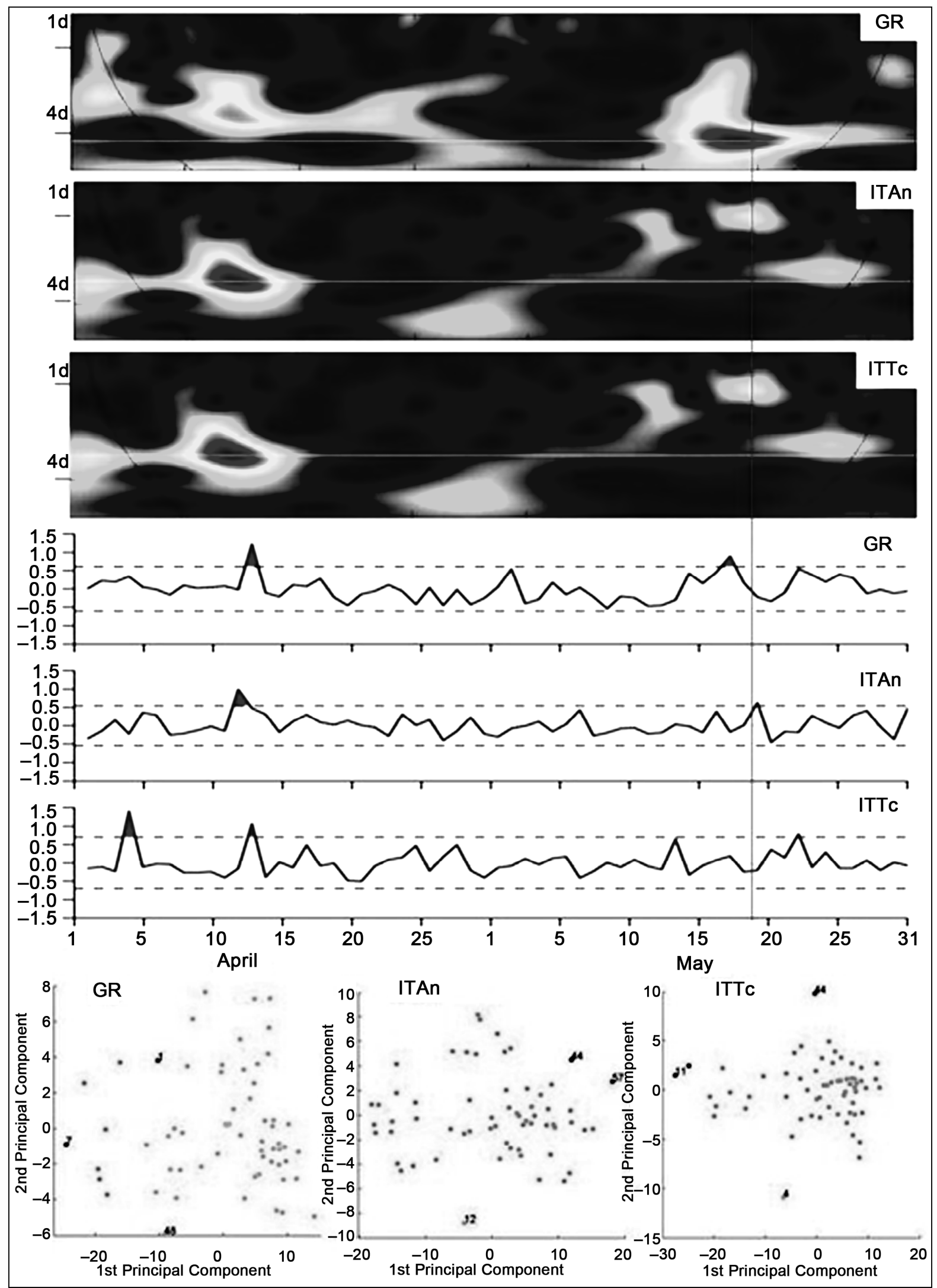

Figure 4. Results of the analysis of the TRT radio data collected by the GR, IT-An and IT-Te receivers during April-May 2011, at night-time. The contents are the same of Figure 3. 
Now, the case reported in Figure 2(b) is considered. Figure 5 shows the results of the analysis of the radio signals collected during April-May 2011 at night-time by the TUR receiver and radiated by the VLF transmitters GBZ and ITS. The 5th Fresnel zone defined by the ITS transmitter and the TUR receiver contains the epicentre of the Turkey earthquake while the other path GBZ-TUR is used as control (Figure 2(b)). As usual, in Figure 5 at the top the Wavelet Spectra, at the middle the Standard Deviation trends and at the bottom the PCA plots, are reported. In this case the three methods are in agreement and reveal: a) The presence of a clear anomaly some days before the occurrence of the earthquake in the ITS data and the absence of this effect in the GBZ data; b) The presence of small disturbance in the last ten days of April and at the beginning of this month in the GBZ data.

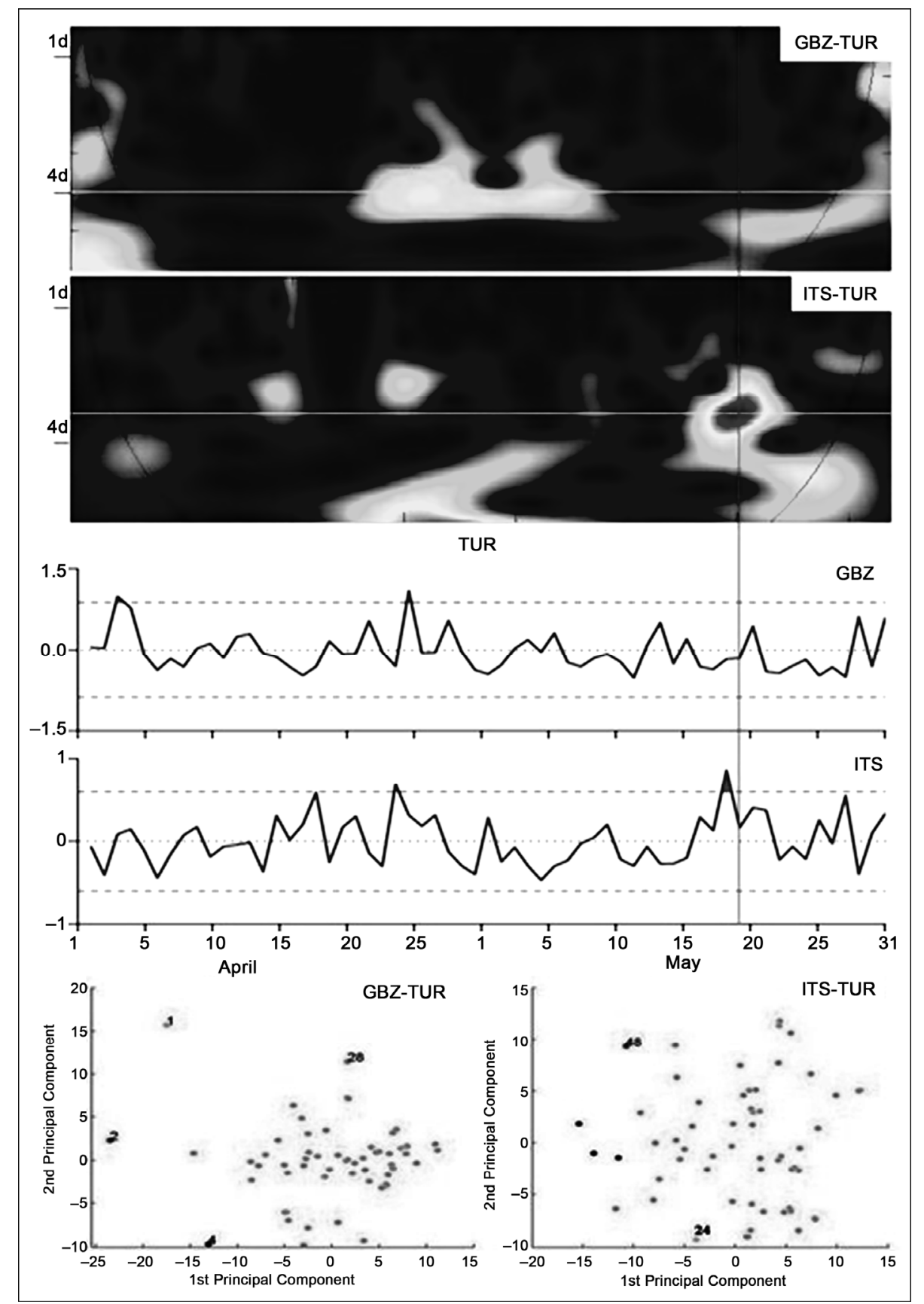

Figure 5. Results of the analysis of the GBZ and ITS radio data collected by the TUR receiver during April-May 2011, at night-time. At the top the Wavelet Spectra, at the middle the Standard Deviation trends and at the bottom the PCA plots. In the Standard Deviation trends the zones over $2 \sigma$ are filled in red; in the PCA plots the numbers on some point (possible outlier) denotes the day counted from April 1. The vertical red line indicates the occurrence of the Turkey earthquake. 
Now the previous results will be discussed. At first we recall the possible genesis of the earthquake precursors [26]:

a) The pre-seismic stress produces an intensification of the micro-fracturing processes and/or changes in existing fissures in the rocks;

b) As a direct consequence of the previous processes, changes occur in the circulation of the underground water and in the mobility of the gases,

$b_{1}$ ) Variations in level/flow and in chemistry of the underground water happen,

$\mathrm{b}_{2}$ ) An afflux of the underground gases (mainly Radon) in the lower atmosphere, perhaps confined in the troposphere, can occur;

c) As a consequence of the processes in the items a) and b), the ground density changes, as well as the ground conductivity and, in some cases, the ground temperature;

d) Electric-magnetic-electromagnetic emissions from the ground can occur in relation to the mentioned processes and these emissions can produce some modifications in the lower atmosphere, again probably confined in the troposphere;

e) As a consequence mainly of the ground density changes reported in the item c), the local gravity changes and gravity waves can be produced as well variations in the atmospheric oscillations (tides, etc.);

f) The previous changes and waves can induce modifications up to the ionosphere;

g) As a consequence of these variations in ionosphere and of the previous variations in the lower atmosphere, disturbances in the propagation of VLF/LF radio signals can be produced.

Now, we can examine the possible seismic effects revealed by our analyses. At first, the results related to the LF data will be discussed.

Both the day-time and night-time data reveal anomalies in all the signals in the first fifteen days of April (Figures 3 and 4). A possible cause [19] could be the meteorological situation in the zone where the transmitter is located. Figure 6 shows the air temperature, the air pressure, the rain and the storms in such zone during April 2011. The meteorological conditions were not so critical and moreover we have noticed that many other times similar conditions happened but no evident disturbance was revealed on the LF radio signals. So, the meteorological justification of the previous anomalies is not convincing. Another possible cause of these anomalies could have been some malfunction of the broadcasting station, but it was ruled out by station manager. So, the possibility that the radio anomalies are connected with the Turkey earthquake of May 11 can be realistic. In such a case these anomalies should be considered a middle term precursor [27] of the earthquake and they could be related to the processes described in the items $\left(b_{2}\right)$ and $(d)$ of the previous synthesis. Then, a clear anomaly appears some days before the occurrence of the earthquake in the TRT radio signal recorded by the GRE receiver at night time (Figure 4). The following statements can be

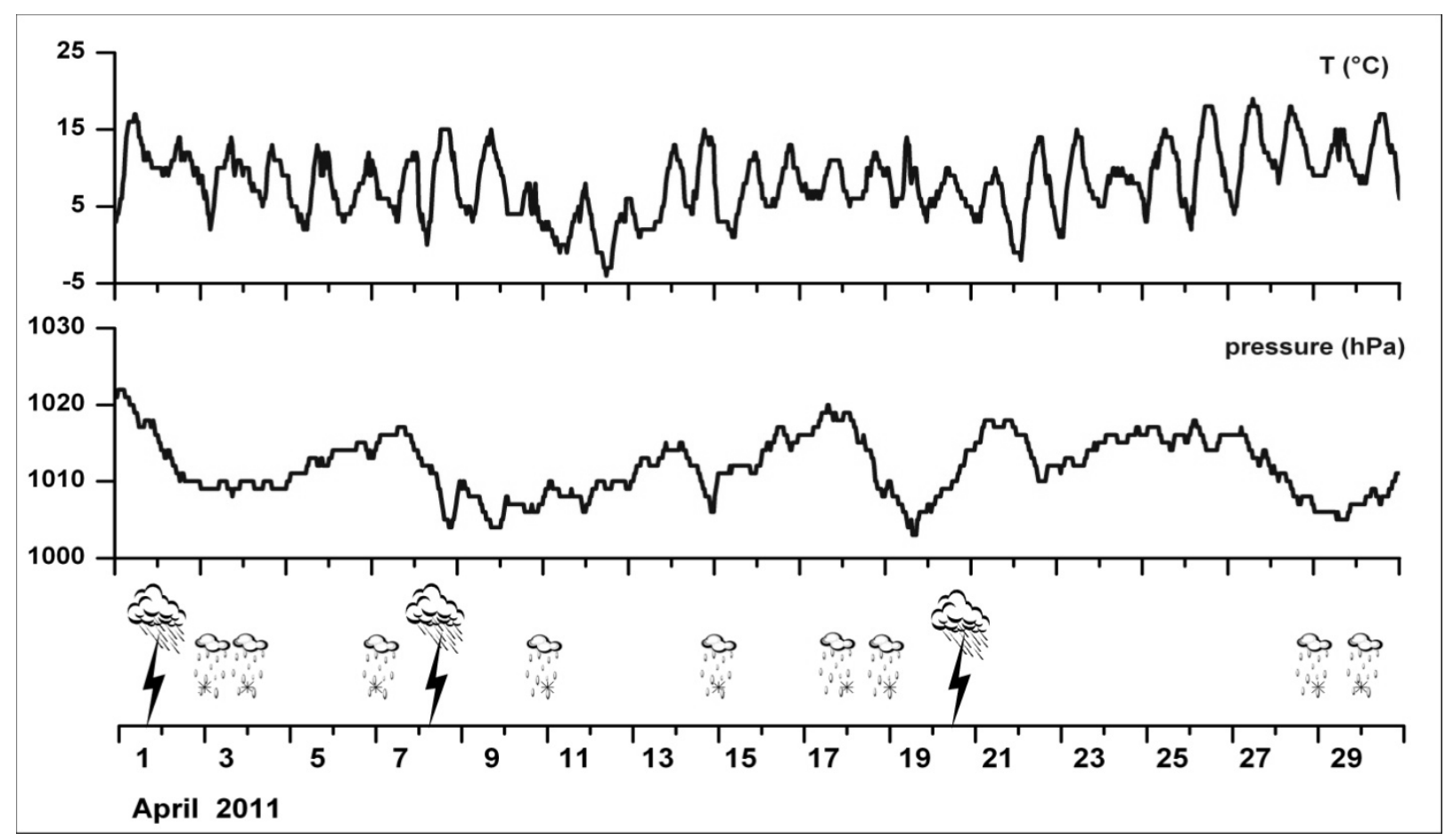

Figure 6. Air temperature and pressure in the zone of the TRT transmitter during April 2011. The rain and the storms are shown, too. 
considered: 1) The other LF signals collected by this receiver coming from Nord and West (Figure 1) do not reveal, in the same period, any disturbance, so the cause of the anomaly is in the East area with respect to the receiver; 2) The anomaly appears only at night time, so it should be related mainly to an ionosphere disturbance; 3 ) the GRE receiver is the nearest (Figure 2(a)) to the TRT transmitter. As a consequence, it seems reasonable to consider the previous anomaly a precursor of the Turkey earthquake, a short term precursor, i.e. related to the final processes [item (g) of the previous synthesis] of the preparation of earthquake.

Now, the results related to the VLF data will be examined. Looking at Figure 5, the main result is a clear anomaly appearing some days before the occurrence of the Turkey earthquake on the ITS night time radio signal collected by the TUR receiver. The GBZ radio signal collected by the same receiver and examined as comparison do not reveal any similar effect. Figure 2(b) shows that the epicentre of the Turkey earthquake is inside the 5th Fresnel zone of the ITS-TUR path, while is out the same zone related to the GBZ-TUR path. So, the possibility that the previous anomaly is a precursor of the Turkey earthquake is consistent. Probably, the anomaly is related to the same disturbance in ionosphere responsible of the LF radio anomaly (the short term one) described in the previous item. Examining together the Figures 2(a) and (b), this statement is convincing.

Finally, from the data here presented, the following remark regarding the efficiency of the different methods of analysis can be made: In order to reveal anomalies in the data sets, the PCA method appears as the most vague; on the contrary the indication of both the Wavelet Spectra and the Standard Deviation trends seems precise and coincident

\section{Conclusion}

This study has confirmed that the VLF and LF radio signals are able to give information on the preparatory phase of earthquakes with $\mathrm{M}_{\mathrm{w}}$ greater than 5.5. In this framework, the earthquake located inside some (5th ad example) Fresnel zone defined by a transmitter and a receiver can produce anomalies, but also those occurring near enough (some hundred kilometres) to a transmitter can do the same. The anomalies are related to disturbances produced in ionosphere, lower atmosphere or both. The Wavelet spectra and the Standard Deviation trends seem valid methods of data analysis for revealing these anomalies. In this study these methods were used on data sets including the anomalies. In order to reveal anomalies in "real time" it is necessary to introduce some modification in the technique of analysis; as for the Wavelet spectra a sufficient number of data, not affected by disturbances, must be added to avoid spurious edge effects and for the Standard Deviation plots, the time interval where the $S D$ mean value is calculated must be defined according to reasonable criteria.

\section{Acknowledgements}

This research was partially supported by Foundation of the Cassa di Risparmio di Puglia bank (F.C.R.P., Bari, Italy).

\section{REFERENCES}

[1] P. F. Biagi, L. Castellana, T. Maggipinto, A. Ermini, G. Perna and V. Capozzi, "Electric Field Strength Analysis of 216 and $270 \mathrm{kHz}$ Broadcast Signals Recorded during 9 Years," Radio Science, Vol. 41, 2006, pp. 3296-3307. doi:10.1029/2005RS003296

[2] P. F. Biagi, R. Piccolo, A. Ermini, S. Martellucci, C. Bellecci, M. Hayakawa, V. Capozzi and S. P. Kingsley, "Possible Earthquake Precursors Revealed by LF Radio Signals," Natural Hazards and Earth System Sciences, Vol. 1, No. 1-2, 2001, pp. 99-104. doi:10.5194/nhess-1-99-2001

[3] P. F. Biagi, R. Piccolo, A. Ermini, S. Martellucci, C. Bellecci, M. Hayakawa and S. P. Kingsley, "Disturbances in LF Radio-Signals as Seismic Precursors," Annali di Geofisica, Vol. 44, No. 5-6, 2001, pp. 1011-1020.

[4] P. F. Biagi and M. Hayakawa, "Possible Premonitory Behaviour of LF Radiowaves on the Occasion of the Slovenia Earthquakes ( $\mathrm{M}=5.2-6.0-5.1)$ Occurred on March-May 1998," In: M. Hayakawa and O. Molchanov, Eds., Seismo Electromagnetics: Lithosphere-AtmosphereIonosphere Coupling, TERRAPUB, Tokyo, 2002, pp. 249253.

[5] P. F. Biagi, L. Castellana, T. Maggipinto, R. Piccolo, A. Minafra, A. Ermini, S. Martellucci, C. Bellecci, G. Perna, V. Capozzi, O. A. Molchanov and M. Hayakawa, "A Possible Preseismic Anomaly in the Ground Wave of a Radio Broadcasting (216 kHz) during July-August 1998 (Italy)," Natural Hazards and Earth System Sciences, Vol. 5, No. 5, 2005, pp. 727-732. doi: 10.5194/nhess-5-727-2005

[6] P. F. Biagi, L. Castellana, T. Maggipinto, R. Piccolo, A. Minafra, A. Ermini, S. Martellucci, C. Bellecci, G. Perna, V. Capozzi, O. A. Molchanov and M. Hayakawa, "LF Radio Anomalies Revealed in Italy by the Wavelet Analysis: Possibile Preseismic Effects during 1997-1998," Physics and Chemistry of the Earth, Vol. 31, No. 4-9, 2006, pp. 403-408. doi:10.1016/i.pce.2005.10.001

[7] P. F. Biagi, L. Castellana, T. Maggipinto, D. Loiacono, V. Augelli, L. Schiavulli, A. Ermini, V. Capozzi, M. S. Solovieva, A. A. Rozhnoi, O. A. Molchanov and M. Hayakawa, "Disturbances in a VLF Radio Signal Prior the $\mathrm{M}=$ 4.7 Offshore Anzio (Central Italy) Earthquake on August 22, 2005," Natural Hazards and Earth System Sciences, Vol. 8, No. 5, 2008, pp. 1041-1048.

doi:10.5194/nhess-8-1041-2008

[8] M. Hayakawa and H. Sato, "Ionospheric Perturbations Associated with Earthquakes, as Detected by Subiono- 
spheric VLF Propagation," In: M. Hayakawa and Y. Fujinawa, Eds., Electromagnetic Phenomena Related to Earthquake Prediction, TERRAPUB, Tokyo, 1994, pp. 391-397.

[9] M. Hayakawa, O. Molchanov, T. Ondoh and E. Kawai, "The Precursory Signature Effect of the Kobe Earthquake on Subionospheric VLF Signals," Journal of Communications Research Laboratory, Vol. 43, 1996, pp. 169-180.

[10] M. Hayakawa, K. Ohta, S. Maekawa, T. Yamauchi, Y. Ida, T. Gotoh, N. Yonaiguchi, H. Sasaki and T. Nakamura, "Electromagnetic Precursors to the 2004 Mid Niigata Prefecture Earthquake," Physics and Chemistry of the Earth, Vol. 31, No. 4-9, 2006, pp. 356-364. doi:10.1016/j.pce.2006.02.023

[11] M. Hayakawa, Y. Kasahara, T. Nakamura, F. Muto, T. Horie, S. Maekawa, Y. Hobara, A. A. Rozhnoi, M. Solovieva and O. A. Molchanov, "A Statistical Study on the Correlation between Lower Ionospheric Perturbations as Seen by Subionospheric VLF/LF Propagation and Earthquakes," Journal of Geophysical Research, Vol. 115, 2010, pp. 15143-15150.

[12] V. A. Morgounov, T. Ondoh and S. Nagai, "Anomalous Variation of VLF Signals Associated with Strong Earthquakes $(\mathrm{M} \geq 7.0)$," In: M. Hayakawa and Y. Fujinawa, Eds., Electromagnetic Phenomena Related to Earthquake Prediction, TERRAPUB, Tokyo, 1994, pp. 409-428.

[13] O. A. Molchanov and M. Hayakawa, "Subionospheric VLF Signal Perturbations Possibly Related to Earthquakes," Journal of Geophysical Research, Vol. 103, No. A8, 1998, pp. 17489-17504. doi:10.1029/98JA00999

[14] O. A. Molchanov, A. Rozhnoi, M. Solovieva, O. Akentieva, J. J. Berthelier, M. Parrot, F. Lefeuvre, P. F. Biagi, L. Castellana and M. Hayakawa, "Global Diagnostic of the Ionospheric Perturbations Related to the Seismic Activity Using the VLF Radio-Signals Collected on the DEMETER Satellite," Natural Hazards and Earth System Sciences, Vol. 6, No. 5, 2006, pp. 745-753. doi:10.5194/nhess-6-745-2006

[15] A. A. Rozhnoi, M. S. Solovieva, O. A. Molchanov and M. Hayakawa, "Middle Latitude LF (40 kHz) Phase Variations Associated with Earthquakes for Quiet and Disturbed Geomagnetic Conditions," Physics and Chemistry of the Earth, Vol. 29, No. 4-9, 2004, pp. 589-598. doi:10.1016/j.pce.2003.08.061

[16] A. A. Rozhnoi, M. S. Solovieva, O. A. Molchanov, M. Hayakawa, S. Maekawa and P. F. Biagi, "Anomalies of LF Signal during Seismic Activity in November-December 2004," Natural Hazards and Earth System Sciences, Vol. 5, No. 5, 2005, pp. 657-660. doi:10.5194/nhess-5-657-2005

[17] A. A. Rozhnoi, M. S. Solovieva, O. A. Molchanov, V. Chebrov, V. Voropaev, M. Hayakawa, S. Maekawa and P. F. Biagi, "Preseismic Anomaly of LF Signal on the Wave Path Japan-Kamchatka during November 2004," Physics and Chemistry of the Earth, Vol. 31, No. 4-9, 2006, pp.
422-427. doi:10.1016/j.pce.2006.02.033

[18] A. A. Rozhnoi, M. S. Solovieva, O. A. Molchanov, V. Gladyshev, O. Akentieva, J. J. Berthelier, M. Parrot, F. Lefeuvre, M. Hayakawa, L. Castellana and P. F. Biagi, "Possible Seismo-Ionosphere Perturbations Revealed by VLF Signals Collected on Ground and on a Satellite," Natural Hazards and Earth System Sciences, Vol. 7, No. 5, 2007, pp. 617-624. doi:10.5194/nhess-7-617-2007

[19] P. F. Biagi, T. Maggipinto, F. Righetti, D. Loiacono, L. Schiavulli, T. Ligonzo, A. Ermini, I. A. Moldovan, A. S. Moldovan, A. Buyuksarac, H. G. Silva, M. Bezzeghoud and M. E. Contadakis, "The European VLF/LF Radio Network to Search for Earthquake Precursors: Setting up and Natural/Man-Made Disturbances," Natural Hazards and Earth System Sciences, Vol. 11, No. 2, 2011, pp. 333341. doi:10.5194/nhess-11-333-2011

[20] K. Schwingenschuh, G. Prattes, B. P. Besser, K. Mocnic, M. Stachel, O. Aydogar, O. Jernei, G. Stangl, M. Y. Boudjada, A. Rozhnoi, M. Solovieva, P. F. Biagi, M. Hayakawa and H. U. Eichelberger, "The Graz Seismo-Electromagnetic VLF Facility," Natural Hazards and Earth System Sciences, Vol. 11, No. 4, 2011, pp. 1121-1127. doi:10.5194/nhess-11-1121-2011

[21] F. Righetti, P. F. Biagi, T. Maggipinto, L. Schiavulli, T. Ligonzo, A. Ermini, I. A. Moldovan, A. S. Moldovan, A. Buyuksarac, H. G. Silva, M. Bezzeghoud, M. E. Contadakis, D. N. Arabelos and T. D. Xenos, "Wavelet Analysis of the LF Radio Signals Collected by the European VLF/LF Network from July 2009 until April 2011," Annals of Geophysics, Vol. 55, No. 1, 2012, pp. 171-180.

[22] I. Daubechies, "Ten Lectures on Wavelets," CBMS-NSF Regional Conferences Series in Applied Mathematics SIAM, Philadelphia, May 1992, pp. 61-80. doi:10.1137/1.9781611970104

[23] G. Strang and T. Nguye, "Wavelets and Filter Banks," Wellesley Cambridge Press, Wellesley, 1996.

[24] C. Torrence and G. P. Compo, "A Practical Guide to Wavelet Analysis," Bulletin of the American Meteorological Society, Vol. 79, No. 1, 1998, pp. 61-78. doi:10.1175/1520-0477(1998)079<0061:APGTWA >2.0. $\underline{\mathrm{CO} ; 2}$

[25] I. T. Joliffe, "Principal Component Analysis," SpringerVerlag, New York, 2002.

[26] P. F. Biagi, "Pre and Post Seismic Disturbances Revealed on the Geochemical Data Collected in Kamchatka (Russia) during the Last 30 Years," In: M. Hayakawa, Ed., Electromagnetic Phenomena Associated with Earthquakes, Transworld Research Network, Kerala, 2009, pp. 97-117.

[27] T. Rikitake, "Earthquake Precursors," Bulletin of Seismological Society of America, Vol. 65, No. 5, 1975, pp. 1133-1162. 